\title{
Mesoscopic modeling of a two-phase flow in the presence of boundaries: The contact angle
}

\author{
R. Benzi, ${ }^{1}$ L. Biferale, ${ }^{1}$ M. Sbragaglia, ${ }^{2}$ S. Succi, ${ }^{3}$ and F. Toschi ${ }^{3,4}$ \\ ${ }^{1}$ Dipartimento di Fisica and INFN, Università di Roma "Tor Vergata," Via della Ricerca Scientifica 1, 00133 Roma, Italy \\ ${ }^{2}$ Department of Applied Physics, University of Twente, P.O. Box 217, 7500 AE Enschede, The Netherlands \\ ${ }^{3}$ Istituto per le Applicazioni del Calcolo CNR, Viale del Policlinico 137, 00161 Roma, Italy \\ ${ }^{4}$ INFN, Sezione di Ferrara, via G. Saragat 1, I-44100, Ferrara, Italy
}

(Received 12 June 2006; published 30 August 2006)

\begin{abstract}
We present a mesoscopic model, based on the Boltzmann equation, for the interaction between a solid wall and a nonideal fluid. We present an analytic derivation of the contact angle in terms of the surface tension between the liquid-gas, the liquid-solid, and the gas-solid phases. We study the dependency of the contact angle on the two free parameters of the model, which determine the interaction between the fluid and the boundaries, i.e. the equivalent of the wall density and of the wall-fluid potential in molecular dynamics studies. We compare the analytical results obtained in the hydrodynamical limit for the density profile and for the surface tension expression with the numerical simulations. We compare also our two-phase approach with some exact results obtained by E. Lauga and H. Stone [J. Fluid. Mech. 489, 55 (2003)] and J. Philip [Z. Angew. Math. Phys. 23, 960 (1972)] for a pure hydrodynamical incompressible fluid based on Navier-Stokes equations with boundary conditions made up of alternating slip and no-slip strips. Finally, we show how to overcome some theoretical limitations connected with the discretized Boltzmann scheme proposed by X. Shan and H. Chen [Phys. Rev. E 49, 2941 (1994)] and we discuss the equivalence between the surface tension defined in terms of the mechanical equilibrium and in terms of the Maxwell construction.
\end{abstract}

DOI: 10.1103/PhysRevE.74.021509

PACS number(s): 83.50.Rp, 05.20.Dd, 68.03.Cd, 68.08.Bc

\section{INTRODUCTION}

The physics of molecular interactions at fluid-solid interfaces is a very active research area with a significant impact on many emerging applications in material science, chemistry, micro and/or nanoengineering, biology, and medicine, see [1-3]. Many problems require the spreading of a liquid on a solid that may either be a simple flat and clean surface or present some degree of roughness contaminated by compounds with different chemical-physical qualities [4]. As for most problems connected with surface effects, fluid-solid interactions become particularly important for micro- and nano-devices, where the physical behavior is largely affected by high surface and/or volume ratios $[5,6]$ whose direct consequence is the enhancement of capillary phenomena with respect to bulk properties $[4,7]$. On the theoretical side, very little is known because of the difficulties to match the classical infinite-volume thermodynamics description with surface effects. From the experimental side the study of the surface properties for the flow-solid interactions is much more difficult than the solid-vacuum case [8-18].

In most cases, to reach quantitative results on specific problems, one is forced to rely on numerical simulations, especially in the presence of complex boundary conditions. To date, two major approaches dominate this field from the numerical side. The first one is based on a pure hydrodynamical description, with the interaction between the flow and the solid fully renormalized in terms of ad hoc boundary conditions for the hydrodynamical fields [19-22]. The main drawback is represented by the difficulty to describe a variety of different solid properties, with complex roughness landscape and chemical-physical attributes. The main advantage is that one can directly focus on spatial and frequency variations up to the typical hydrodynamical scales.
The second approach attacks the problem from an atomistic description, by integrating the Newton equations for a set of molecules interacting via a Lennard-Jones potential. This is the basic idea behind molecular dynamics (MD), which requires an additional ad hoc tuning of the free parameters entering the potential between liquid-liquid molecules and between liquid-solid molecules [22-27]. These parameters are fine tuned by comparison with the experiments and are mainly of two types: the overall strength of the interactions and the typical interacting distance (fixed by the relative weight between the attractive and repulsive terms). The main drawback is here represented by the congenital scale separation between this method and continuum phenomena (see [28]) and consequently the inability to describe spatial fluctuations on scales which are much larger than the intermolecular interactions and temporal fluctuations larger than a few milliseconds. The advantage is given by its apparent $a b$ initio nature, although to be of any practical use, the method needs to be supplemented with experimental data $[29,30]$. In this paper we follow a third route, focusing on a mesoscopic modeling of the solid-liquid interaction based on the kinetic theory of dense fluids [31,32].

The method, known as lattice Boltzmann equation (LBE), directly accesses spatial and temporal fluctuations at the hydrodynamical level with the extra bonus of a large flexibility in the description of the chemical and physical properties of the boundary conditions (see [33-35] and for exhaustive reviews see $[36,37])$. With respect to the MD approach, one pays the price to move the matching with the experimental data to the level of bulk interaction between the Boltzmann distribution function (the equivalent of liquid-liquid MD potential) and to the boundary conditions imposed on the Boltzmann equation (the equivalent of the solid-liquid potential in the MD). Ideally one would supply the infinite Bogoliubov-Born-Green-Kirkwood-Yvon (BBGKY) hierar- 
chy $[38,39]$ typical of any kinetic description, with atomistic information, thereby closing the problem without any approximation. In most cases, a more practical approach is taken: in order to derive useful kinetic description, some educated guess on the many-body BBGKY hierarchy are proposed and tested a posteriori.

In this paper, we shall focus on surface effects in the presence of phase coexistence between a liquid and its saturated vapor. In particular, we aim at investigating how to develop an effective mesoscopic description of the surface tension between the liquid-gas, $\gamma_{l g}$, the liquid-solid, $\gamma_{l s}$ the gas-solid $\gamma_{g s}$ phases and, more practically of the contact angle $\theta[4,7]$ that can be defined from the above surface tensions:

$$
\cos (\theta)=\frac{\gamma_{g s}-\gamma_{l s}}{\gamma_{l g}}
$$

We will perform an analytical and numerical study within the mean field method proposed by $[40,41]$, based on a lattice Boltzmann equation with an effective two-body potential described only in terms of local, single molecule, properties of the fluid (see the next section for a detailed description of the method). The model provides, in our opinion, the simplest coherent description of the many-body interaction typical of dense fluids within the lattice Boltzmann equation framework for non-ideal fluids [40-44]. Other important attempts were already published by [45-47] concerning a lattice transcription of mean field thermodynamic boundary conditions [48] in the framework of free-energy multiphase methods (see [42]). Numerical investigations of the contact angle within a LBE approach were also presented by $[44,49]$ but without any analytical control on the links with the surface tension as proposed here.

In this paper, we review first the method as defined by $[40,41]$ for bulk flows (no boundaries) and we extend it to include the interaction with a given solid surface by the introduction of suitable boundary conditions. This defines a theoretical scheme able to incorporate nonideal effects (phase transitions) triggered by the presence of the solid boundary and complex fluid properties connected to the actual density profiles (contact lines, contact angle, capillary phenomena, surface tensions, etc.). The main result of the first part is an exact analytical expression of the contact angle in terms of the surface tensions derived from the hydrodynamical limit of the Boltzmann equations. In the second part, we perform a systematic study of the contact angle dependency on the boundary properties and we compare our two-phase approach with some exact results obtained for a pure hydrodynamical single-phase fluid based on NavierStokes equations with suitable boundary conditions $[19,20]$. We also show how to overcome some theoretical limitations connected with the discretized Boltzmann scheme here utilized, proposed in [40], and we discuss the equivalence between the surface tension defined in terms of the mechanical equilibrium or in terms of the thermodynamical "Maxwell construction" [50,51]. Finally, we discuss the possible application of this method to describe non-stationary flows in microchannels, the apparent slip phenomenon, and the wetting and/or dewetting transition induced by microcorrugation in the boundaries.

\section{THE SHAN-CHEN (SC) APPROACH TO NONIDEAL FLUIDS: THE INCLUSION OF BOUNDARIES}

As soon as one goes beyond the "ideal gas" description, allowing for phase transition and for density and temperature variations inside the flow, the correct way to approach the kinetic problem is to start from the BBGKY formalism [39]. Phase transitions are triggered by critical dependency of the thermodynamic variables on small variations in the local density, temperature, and pressure fields. In order to describe such phenomena, one needs to go beyond the description based on the probability density to observe a single molecule with a given velocity, $\boldsymbol{v}_{1}$, at position $\boldsymbol{r}_{1}$ and at time $t$, $f_{1}\left(\boldsymbol{r}_{1}, \boldsymbol{v}_{1}, t\right)$. In particular, one needs to consider at least the two-particle distribution, $f_{1,2}\left(\boldsymbol{r}_{1}, \boldsymbol{v}_{1}, \boldsymbol{r}_{2}, \mathbf{v}_{2}, t\right)$, which explicitly enters in the Boltzmann equation via the collisional term, $\Omega$ :

$$
\partial_{t} f_{1}+\boldsymbol{v}_{1} \cdot \partial_{\mathbf{r}_{1}} f_{1}+\mathbf{K}_{1} \cdot \partial_{\boldsymbol{v}_{1}} f_{1}=\Omega,
$$

where $\mathbf{K}_{1}$ is an external body force and

$$
\Omega=-\int d \boldsymbol{v}_{2} d \boldsymbol{r}_{2} \partial_{\boldsymbol{v}_{1}} f_{1,2} \partial_{\boldsymbol{r}_{1}} V\left(r_{12}\right)
$$

with $V\left(r_{12}\right)=V\left(\left|\boldsymbol{r}_{1}-\boldsymbol{r}_{2}\right|\right)$ being the interparticle potential. The BBGKY hierarchy prescribes the evolution of $f_{1,2}$ in terms of the three-particle densities, $f_{1,2,3}$, the evolution of $f_{1,2,3}$ in terms of the four-particles density and so on. The simplest closure which takes into account the two-particles interaction consists in adopting a "mean-field" approach for the collisional terms. This approach starts by rewriting $f_{1,2}$ in the equivalent form:

$$
f_{1,2}\left(\boldsymbol{r}_{1}, \boldsymbol{v}_{1}, \boldsymbol{r}_{2}, \boldsymbol{v}_{2}\right)=f_{1}\left(\boldsymbol{r}_{1}, \boldsymbol{v}_{1}\right) f_{2}\left(\boldsymbol{r}_{2}, \boldsymbol{v}_{2}\right) g\left(\boldsymbol{r}_{1}, \boldsymbol{r}_{2}, \boldsymbol{v}_{1}, \boldsymbol{v}_{2}\right),
$$

where we have introduced the two-particles correlation function, $g\left(\boldsymbol{r}_{1}, \boldsymbol{r}_{2}, \boldsymbol{v}_{1}, \boldsymbol{v}_{2}\right)$. To proceed further one needs to make some approximation on the two-body correlation function $g\left(\boldsymbol{r}_{1}, \boldsymbol{r}_{2}, \boldsymbol{v}_{1}, \boldsymbol{v}_{2}\right)$. The celebrated "molecular chaos" assumption of Boltzmann gives $g=1$, i.e., absence of both velocity and spatial correlations [32]. In the less restrictive case where only velocity correlations vanish, one has

$$
g\left(\boldsymbol{r}_{1}, \boldsymbol{r}_{2}, \boldsymbol{v}_{1}, \boldsymbol{v}_{2}\right)=g\left(\boldsymbol{r}_{1}, \boldsymbol{r}_{2}\right)
$$

and the collisional term can be (see also [52]) rewritten as

$$
\Omega=-\partial_{\boldsymbol{v}_{1}} f_{1} \int d \boldsymbol{r}_{2} \rho\left(\boldsymbol{r}_{2}\right) g\left(\boldsymbol{r}_{1}, \boldsymbol{r}_{2}\right) \partial_{\mathbf{r}_{1}} V\left(r_{12}\right),
$$

where we have used the definition of the local density as

$$
\rho(\boldsymbol{r}, t)=\int d \boldsymbol{v} f(\boldsymbol{r}, \boldsymbol{v}, t) .
$$

The approximation (4) is at the core of many lattice Boltzmann descriptions of nonideal fluids because now the collisional term has the form of a body force term, 
$\Omega \propto \mathbf{K}_{1} \cdot \partial_{\boldsymbol{v}_{1}} f_{1}$, and may be seen as a renormalization of the local pressure tensor via the introduction of nonideal terms in the equation of state $[36,53]$.

More quantitatively, if we start from Eq. (2) and together with Eq. (5) we define the local momentum as

$$
\rho \boldsymbol{u}(\boldsymbol{r}, t)=\int d \boldsymbol{v} f(\boldsymbol{r}, \boldsymbol{v}, t) \boldsymbol{v}
$$

we obtain (see $[39,52]$ ) conservative equations for the two local fields:

$$
\begin{gathered}
\partial_{t} \rho+\nabla \cdot(\rho \boldsymbol{u})=0, \\
\partial_{t}(\rho \boldsymbol{u})+\nabla \cdot\left(\int d \boldsymbol{v} f \boldsymbol{v} \boldsymbol{v}\right)=\int d \boldsymbol{v} \Omega \boldsymbol{v}=-\nabla \stackrel{\leftrightarrow}{W},
\end{gathered}
$$

where the tensor $\stackrel{\leftrightarrow}{W}$ is directly related to the interaction potential $V\left(r_{12}\right)$ :

$$
\begin{aligned}
W_{i, j}= & -\frac{1}{2} \int d \boldsymbol{r}_{1} d \boldsymbol{v}_{1} d \boldsymbol{v}_{2} d \boldsymbol{r}_{2} f_{1} f_{2} g \\
& \times \delta\left(\boldsymbol{r}-\boldsymbol{r}_{1}\right) V^{\prime}\left(r_{12}\right) r_{12}^{-1}\left(r_{12}\right)_{i}\left(r_{12}\right)_{j} .
\end{aligned}
$$

The previous equations are clearly locally conservative for density and globally conservative for momentum [39].

In the realm of Boltzmann equations, a popular way to simplify the collisional integral is to write it as a simple relaxation term (with characteristic time $\tau$ ) towards a suitable local equilibrium. This is the celebrated BGK approximation given by [54] and one may wonder which is the simplest BGK description consistent with Eqs. (7) and (8). By consistent BGK description, we mean a single time relaxation collisional term of the form

$$
\Omega_{B G K}=-\frac{1}{\tau}\left(f-f^{(M)}\right),
$$

where the equilibrium distribution $f^{(M)}$ is the local Maxwellian in $D$ dimensions:

$$
f^{(M)}=\frac{\rho^{\prime}}{\left(2 \pi K T^{\prime}\right)^{D / 2}} \exp \left\{\frac{\left(\boldsymbol{v}-\boldsymbol{u}^{\prime}\right)^{2}}{2 K T^{\prime}}\right\}
$$

and the parameters $\rho^{\prime}(\boldsymbol{r}, t), \boldsymbol{u}^{\prime}(\boldsymbol{r}, t), T^{\prime}(\boldsymbol{r}, t)$ can be chosen in such a way to be consistent with global balance equations. In particular, straightforward Gaussian integration yields to $\rho^{\prime}(\boldsymbol{r}, t)=\rho(\boldsymbol{r}, t)$ and

$$
\boldsymbol{u}^{\prime}(\boldsymbol{r}, t)=\boldsymbol{u}(\boldsymbol{r}, t)-\frac{1}{\tau \rho} \boldsymbol{\nabla} \stackrel{\leftrightarrow}{W}(\boldsymbol{r}, t)
$$

which means a space-time dependent shift of the mean value of the local Maxwellian. The Shan-Chen model $[40,41]$ is precisely equivalent to such an approach with the assumption

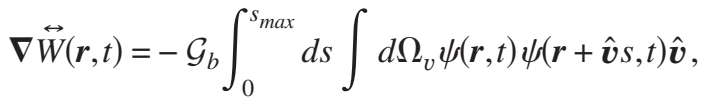

where with $d \Omega_{v}$ we denote the integration over the angular dependency of the unit vector, $\hat{\boldsymbol{v}}$ and where $\psi(\boldsymbol{r}, t)$ is a meanfield potential which depends on $\boldsymbol{r}$ and $t$ only through the local density, i.e., $\psi(\boldsymbol{r}, t) \equiv \psi(\rho(\boldsymbol{r}, t))$. In the above, $\left|s_{\max } \hat{\boldsymbol{v}}\right|$ represents the range of the interactions and $\mathcal{G}_{b}$ a coupling constant, something like an inverse temperature for the model.

In principle, the temperature $T^{\prime}(\boldsymbol{r}, t)$ in (11) should also be changed on account of thermodynamics consistency and total energy conserving dynamics (potential plus kinetic). However, being interested in isothermal phenomena, we keep $K T^{\prime}=c_{s}^{2}, c_{s}^{2}$ being the sound speed velocity (for a possible extension of the model to include also temperature fluctuations see [52]).

Upon discretization of the approximation described before, we derive immediately the (lattice) Boltzmann equations $[55,56]$ as follows:

$$
\begin{aligned}
f_{\alpha}(\boldsymbol{x} & \left.+\boldsymbol{c}_{i} \Delta t, t+\Delta t\right)-f_{\alpha}(\boldsymbol{x}, t) \\
& =-\frac{\Delta t}{\tau}\left\{f_{\alpha}(\boldsymbol{x}, t)-f_{\alpha}^{(e q)}\left[\rho(\boldsymbol{x}, t), \boldsymbol{u}^{\prime}(\boldsymbol{x}, t)\right]\right\},
\end{aligned}
$$

where $\boldsymbol{x}$ runs on a two- (or three-) dimensional lattice and $\Delta t=1$ is the time stepping in the numerical scheme. The LHS of (14) is the molecular free streeming of a discrete set $\left(\boldsymbol{c}_{\alpha}, \alpha=0, \ldots, N\right)$ of velocities whereas the right-hand side represents molecular collisions via a simple relaxation towards the local equilibrium $f_{\alpha}^{(e q)}$ (the local Maxwellian expanded to second order in the Mach number) in a time lapse of the order of $\tau$. This relaxation time fixes the fluid kinematic viscosity as $\nu=c_{s}^{2}\left(\tau-\frac{1}{2}\right)$ where $c_{s}=1 / \sqrt{3}$ in the present work (see [56]). The fluid density and momentum are given by

$$
\rho=\sum_{\alpha} f_{\alpha}, \quad \boldsymbol{u}=\frac{1}{\rho} \sum_{\alpha} f_{\alpha} \boldsymbol{c}_{\alpha}
$$

and can be shown to evolve according to the Navier-Stokes equations of fluid-dynamics, [56]:

$$
\begin{gathered}
\rho\left[\partial_{t} \boldsymbol{u}+(\boldsymbol{u} \cdot \nabla) \boldsymbol{u}\right]=-\nabla \stackrel{\leftrightarrow}{P}_{0}+\boldsymbol{F}+\nabla \cdot(\nu \rho \nabla \boldsymbol{u}), \\
\partial_{t} \rho+\nabla \cdot(\rho \boldsymbol{u})=0,
\end{gathered}
$$

being $\stackrel{\leftrightarrow}{P}_{0}$ the ideal pressure tensor given by the perfect-gas equation of state: $\stackrel{\leftrightarrow}{P_{0}}=\overleftrightarrow{I} c_{s}^{2} \rho$, where $\stackrel{\leftrightarrow}{I}$ is the unit tensor. Nonideal effects are modeled through the self-consistent body force term (13) that can be discretized as

$$
\boldsymbol{F}(\boldsymbol{x}, t)=-\mathcal{G}_{b} \psi(\boldsymbol{x}, t) \sum_{\alpha} w_{\alpha} \psi\left(\boldsymbol{x}+\boldsymbol{c}_{\alpha} \Delta t, t\right) \boldsymbol{c}_{\alpha},
$$

where $\psi(\boldsymbol{x}, t)$ is the lattice version of the mean-field potential previously used and $w_{\alpha}$ are normalization weights (see Appendix A for more technical details). Due to this body force at each time step we consistently redefine the velocity $\boldsymbol{u}^{\prime}$ in the equilibrium distribution $f^{(e q)}$ as

$$
\boldsymbol{u}^{\prime}=\frac{1}{\rho} \sum_{\alpha} \boldsymbol{c}_{\alpha} f_{\alpha}-\frac{1}{\tau \rho} \mathcal{G}_{b} \psi(\boldsymbol{x}, t) \sum_{\alpha} \psi\left(\boldsymbol{x}+\boldsymbol{c}_{\alpha} \Delta t, t\right) \boldsymbol{c}_{\alpha}
$$

which in turns implies a nondiagonal pressure tensor $\stackrel{\leftrightarrow}{P}$ deduced from the condition: 


$$
-\nabla \stackrel{\leftrightarrow}{P}+\nabla \stackrel{\leftrightarrow}{P}_{0}=F
$$

By expanding in Taylor series the interparticle potential one gets (see Appendix A) in the case $\Delta t=1$ :

$$
\begin{aligned}
P_{i j}= & {\left[c_{s}^{2} \rho+\frac{1}{2} c_{s}^{2} \mathcal{G}_{b} \psi^{2}+\frac{1}{2} c_{s}^{4} \mathcal{G}_{b} \psi \Delta \psi+\frac{\mathcal{G}_{b} c_{s}^{4}}{4}|\nabla \psi|^{2}\right] \delta_{i j} } \\
& -\frac{1}{2} c_{s}^{4} \mathcal{G}_{b} \partial_{i} \psi \partial_{j} \psi
\end{aligned}
$$

Let us note that there is always a certain degree of arbitrariness in deriving the full expression of the pressure tensor in the continuum limit [17]. The most reasonable way to do it is to impose that the constraint (17) is verified up to the second order in the Taylor expansion of both pressure and forcing expression. We stop at second order because Navier-Stokes equations are obtained from the LBE at the second order in the Chapman Enskog expansion. The above expression for the pressure tensor is different from the one proposed in Eq. (19) of [40]. The reason is that in [40] the constraint (17) is verified only up to the first order in Taylor expansion. This difference is important for the thermodynamic consistency of isothermal flow as it will be described in Sec. II D, see also [43].

The first two terms in the diagonal part of (18) describe the bulk homogeneous phase transition by the nonideal equation of state:

$$
P_{b}(\rho)=c_{s}^{2} \rho+\frac{1}{2} c_{s}^{2} \mathcal{G}_{b} \psi^{2}(\rho) .
$$

with a typically used functional form:

$$
\psi(\rho)=\left(1-e^{-\rho / \rho_{0}}\right)
$$

with $\rho_{0}$ a reference density.

Now, we will consider a general background that is independent of the functional forms of $\psi$, given for granted that, for a quantitative agreement with MD and experiments, one can choose different functional forms and/or different interparticle interactions. We will be back on the importance of the functional form later on in Sec. II D, where we investigate the Maxwell construction in the $(P, V)$ diagram of the model. Let us only discuss for the moment the qualitative behavior imposed on the $\rho$, dependency of $\psi(\rho)$ by two physical constraints. First, for small densities $\rho$ we need to recover the equation of state of an ideal gas, which requires that

$$
\psi(\rho) \propto \rho, \quad \rho \rightarrow 0 .
$$

Second, for large local densities, the interacting potential must saturate:

$$
\psi(\rho) \rightarrow \text { const. }, \quad \rho \gg \rho_{0}
$$

a requirement meant to mimic the hard-core properties of real molecules which prevent unphysical density accumulations. Any smooth functional form which satisfies the two previous requirements leads to a phase transition as soon as $\mathcal{G}_{b}$ becomes smaller than a critical value $\mathcal{G}_{c}$ (with $\mathcal{G}_{c}$ being negative), where the fluid starts to exhibit two coexisting phases with the same pressure. All the other terms in Eq. (18), which depend explicitly on the density variations, de- scribe the development of an interface profile with its own surface tension (as soon as the isotropy of the pressure tensor is violated).

\section{A. SC model without boundaries}

For the sake of completeness, let us summarize again the steps needed to calculate the density profile in presence of a liquid-gas interface in an unbounded domain as shown for the first time by [40]. This calculation will be used later on to implement the expression of the contact angle in presence of boundaries. In order to calculate the density profile we need to use the general expression of the pressure tensor (18) and insert it into the mechanical equilibrium condition:

$$
\boldsymbol{\nabla} \stackrel{\leftrightarrow}{P}(\boldsymbol{x})=0,
$$

with the appropriate boundary conditions $\rho(-\infty)=\rho_{g}$ and $\rho(+\infty)=\rho_{l}$ for a planar interface between liquid and gas. Let us suppose that the interface develops along the $y$ coordinate. Under this geometry, the pressure tensor becomes anisotropic with a mismatch between the transverse components $P_{x x}(y)$ $=P_{z z}(y)$ and the normal component $P_{y y}(y)$. The condition that the interface does not move, (21), implies that the normal component remains constant and equal to the value in the bulk, $P_{\text {bulk }}$, throughout the interface:

$$
P_{y y}(y) \equiv P_{b u l k}=c_{s}^{2} \rho+\frac{1}{2} c_{s}^{2} \mathcal{G}_{b} \psi^{2}+\frac{1}{2} c_{s}^{4} \mathcal{G}_{b} \psi \partial_{y y} \psi-\frac{\mathcal{G}_{b} c_{s}^{4}}{4}\left|\partial_{y} \psi\right|^{2} .
$$

The density shape is now fully determined by solving (22) with the requirement that the liquid and gas phase share the same value of the bulk pressure:

$$
P_{\text {bulk }}=c_{s}^{2} \rho_{g}+\frac{1}{2} c_{s}^{2} \mathcal{G}_{b} \psi^{2}\left(\rho_{g}\right)=c_{s}^{2} \rho_{l}+\frac{1}{2} c_{s}^{2} \mathcal{G}_{b} \psi^{2}\left(\rho_{l}\right) .
$$

By making the change of variables $(d \rho / d y)^{2}=z$, one may rewrite the mechanical equilibrium as an ordinary differential equation for the interparticle potential where only derivatives with respect to the density $\rho$ appear:

$$
P_{b u l k}=c_{s}^{2} \rho+\frac{\mathcal{G}_{b}}{2} c_{s}^{2} \psi^{2}+\frac{\mathcal{G}_{b} c_{s}^{4}}{2}\left\{\frac{1}{2} \frac{\psi^{2}}{\psi^{\prime}} \frac{d}{d \rho}\left[\left(\frac{d \rho}{d y}\right)^{2}\left(\frac{d \psi}{d \rho}\right)^{2} \frac{1}{\psi}\right]\right\} .
$$

The above differential equation can be integrated explicitly to give

$$
z(\rho)=\frac{4 \psi}{\mathcal{G}_{b} c_{s}^{4}\left(\psi^{\prime}\right)^{2}} \int_{\rho_{g}}^{\rho}\left(P_{b u l k}-c_{s}^{2} \rho-\frac{\mathcal{G}_{b}}{2} c_{s}^{2} \psi^{2}\right) \frac{\psi^{\prime}}{\psi^{2}} d \rho
$$

with $\psi^{\prime}=\partial \psi / \partial \rho$. If the two extremes of the integral are chosen inside the bulk phases we get $z(\rho)=0$ for $\rho=\rho_{l}$ and $\rho$ $=\rho_{g}$. It is easy to realize that in order to be compatible with the latter boundary conditions, we must require

$$
\int_{\rho_{l}}^{\rho_{g}}\left[P_{b u l k}-c_{s}^{2} \rho-\frac{c_{s}^{2} \mathcal{G}_{b}}{2} \psi(\rho)^{2}\right] \frac{\psi^{\prime}}{\psi^{2}} d \rho=0
$$

which fixes, together with (23), the two densities $\rho_{l}$ and $\rho_{g}$ as shown by [40]. 
The whole profile can be obtained by inverting (25) or by directly numerically solving (24) starting from the inside of one bulk phase and making a small initial spatial perturbation on the constant density profile. Let us note that Eqs. (22), (25), and (26) are different from Eqs. (21), (24), and (25) of [40] because of the different requirements imposed here in the derivation of the pressure tensor as discussed after Eq. (18).

\section{B. Surface tension and contact angle}

Following [7], we define the liquid-gas surface tension $\gamma_{l g}$ as the integral along the coordinate normal to the interface of the mismatch between normal and transverse components of the above tensor. More precisely, assuming that the only dependence is in the $y$ coordinate, the local increment of the surface tension is

$$
P_{y y}-P_{x x}=\frac{d \gamma_{l g}}{d y}=-\frac{1}{2} c_{s}^{4} \mathcal{G}_{b}\left|\partial_{y} \psi\right|^{2} .
$$

Upon integration from $-\infty$ to $+\infty$, the surface tension is readily calculated:

$$
\gamma_{l g}=-\frac{1}{2} c_{s}^{4} \mathcal{G}_{b} \int_{-\infty}^{+\infty}\left|\partial_{y} \psi\right|^{2} d y .
$$

This implies the existence of transverse stresses that would result in a pressure drop for spherical interfaces and in the most general Laplace law for a curved surface:

$$
\Delta p=\gamma_{l g}\left(\frac{1}{R_{1}}+\frac{1}{R_{2}}\right)
$$

being $R_{1}$ and $R_{2}$ the local principal radii of curvature of the surface, see $[4,7,40]$. Let us now discuss how to generalize the previous results to the case of a solid boundary. In our language, mechanical equilibrium between multiphase systems (say liquid, gas, and solid) can be formulated as a more general problem. We want to study the mechanical equilibrium by imposing that the density profile must match some given value at the boundary position:

$$
\begin{gathered}
\nabla \stackrel{\leftrightarrow}{P}(\boldsymbol{x})=0, \\
\psi\left(\rho\left(\boldsymbol{x}_{w}\right)\right)=\psi\left(\rho_{w}\right),
\end{gathered}
$$

where the solid wall is at position $\boldsymbol{x}_{w}$. Equation (30) is nothing but the mechanical equilibrium of a multiphase system in the presence of a boundary condition. The value of the interparticle potential at the wall, $\psi\left(\rho_{w}\right)$, is a free parameter in the model, and it is not meant to be related with the "true" density of the solid phase. It will be used here to tune different wall properties.

Focusing, for the sake of simplicity, on two-dimensional systems (see Fig. 12 in Appendix B) the mechanical equilibrium equation translates into

$$
\partial_{x} P_{x x}+\partial_{y} P_{x y}=0
$$

or equivalently $\left(\partial_{x} P_{x y}+\partial_{y} P_{y y}=0\right)$, whose meaning is that the flux over an arbitrary contour (Green's theorem) of the vec- tor $\left(P_{x x}, P_{x y}\right)$ is zero. With reference to Fig. 12 in Appendix $\mathrm{B}$ we note that for such a calculation we need to specify the pressure tensor along a solid-gas interface and liquid-solid interface. If we choose the rectangular contour shown in Fig. 12 and impose the flux of the above vector exactly to zero (details are given in Appendix B) we obtain:

$$
\cos (\theta)=\frac{\int_{s g}\left|\partial_{y} \psi\right|^{2} d y-\int_{s l}\left|\partial_{y} \psi\right|^{2} d y}{\int_{l g}\left|\partial_{y} \psi\right|^{2} d y},
$$

where $\int_{s l}\left|\partial_{y} \psi\right|^{2} d y, \int_{s g}\left|\partial_{y} \psi\right|^{2} d y, \int_{l g}\left|\partial_{y} \psi\right|^{2} d y$ indicate the positive integrals calculated along the solid-liquid, solid-gas, and liquid-gas interfaces.

From the above relation (32), which fully determines the contact angle in our LBE scheme, one naturally extracts the definition of the surface tensions:

$$
\gamma_{\alpha, \beta}=-\frac{1}{2} c_{s}^{4} \mathcal{G}_{b} \int_{\alpha, \beta}\left|\partial_{y} \psi\right|^{2} d y,
$$

where with $\alpha, \beta$ we mean any two among the liquid, gas, and solid phases. Note that, rigorously speaking, the surface tensions between liquid-solid and between gas-solid are defined only modulo as an additive constant, being operationally defined in terms of the contact angle which depends only on their difference [4]. The above definition is consistent with the requirement to have $\gamma_{l s}=0$ when the wall has the same density of the liquid (perfect wetting). Correspondingly, one may also imagine a situation when the gas phase perfectly matches the wall properties, $\rho_{g}=\rho_{w}$, where we have $\gamma_{s g}=0$ and consequently a complete dewetting.

\section{Analytical and numerical results}

From the mechanical equilibrium condition (30), we may calculate the density variation along an interface between any two of the three phases, generalizing the calculation made by [40] for the gas-liquid interface only. To accomplish this, we must integrate the equation for the normal component of the pressure tensor (22) imposing the boundary conditions at the solid, $\rho\left(y=y_{w}\right)=\rho_{w}$, and $\rho(\infty)=\rho_{\beta}$ where with $\beta$ we denote either the liquid or the gas phase (see also Sec. II D for more details). In Fig. 1 we show two such profiles. In our first case at the center of the channel there is only liquid and the gas phase cannot develop, i.e., the averaged density is larger than the liquid density at coexistence, while in the second case the averaged density is chosen such that a gas phase can develop between the liquid and the wall.

Once the density profile is known, it is easy to obtain the corresponding surface tension by plugging the density profile into the expression (33). For example, in Fig. 2, we show the surface tensions, $\gamma_{l s}, \gamma_{l g}, \gamma_{s g}$ at a given temperature $\left(\mathcal{G}_{b}\right)$ and by changing $\rho_{w}$, i.e., by varying the wetting properties of the surface. Of course, only the first two will depend on $\rho_{w}$ and, accordingly to our previous discussion, they must satisfy $\gamma_{l s}=0$ for $\rho_{w}=\rho_{l}$ (perfect wetting) and $\gamma_{g s}=0$ for $\rho_{w}=\rho_{g}$.

Given the surface tensions for a fixed temperature one may readily calculate the contact angle which describes the 


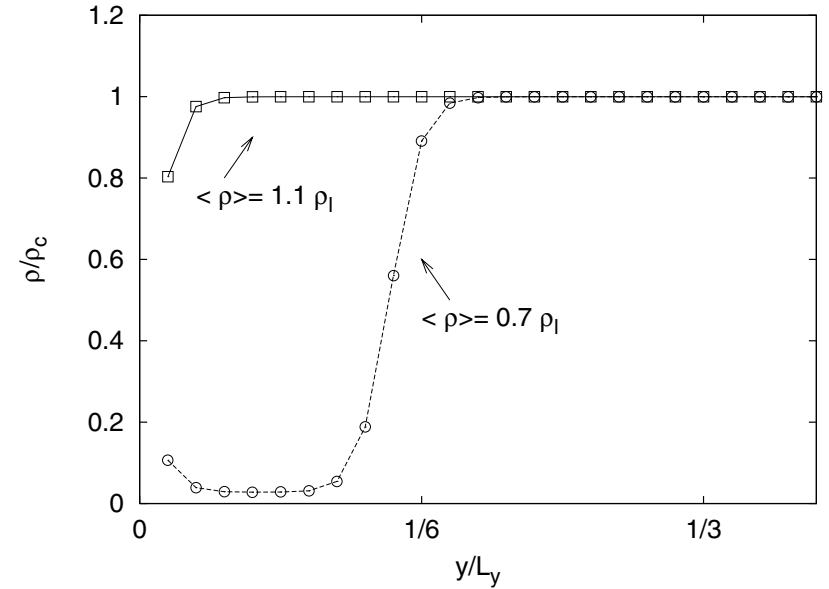

FIG. 1. The stationary state configuration for the density field as a function of the relative distance from the wall. We present the density field normalized to the center channel density for two different values of the average density of the system: $\langle\rho\rangle=1.1 \rho_{l}(\square)$ and $\langle\rho\rangle=0.7 \rho_{l}(\bigcirc)$. In both cases we integrate numerically the lattice Boltzmann equation with $\tau=1$ in a $2 d$ channel with two walls (bottom and lower) and a periodic boundary condition in the stream-wise direction. The dimensions of the channel are $L_{x} \times L_{y}$ $=80 \times 45$ and $\mathcal{G}_{b}=-6.0$, being $\mathcal{G}_{c}=-4.0$ the critical point of the system. The liquid and gas density for this value are, respectively, $\rho_{l}=2.65$ and $\rho_{g}=0.07$ in lattice Boltzmann units. Note that the density tends to match a given value at the wall (for $y / L_{y}=0$ ) which is different from both liquid and gas values; this is because of the chosen boundary condition, $\psi\left(\rho_{w}=0.5\right)$.

macroscopic properties of the surface as a function of the mesoscopic boundary condition imposed on $\psi\left(\boldsymbol{x}_{w}\right)=\psi\left(\rho_{w}\right)$. This is an important methodological result of this paper, i.e., we provide a mesoscopic way to parametrize the hydrodynamical behavior of fluid in the proximity of a surface (with different contact angles) as a function of the tunable free parameter $\psi\left(\rho_{w}\right)$.

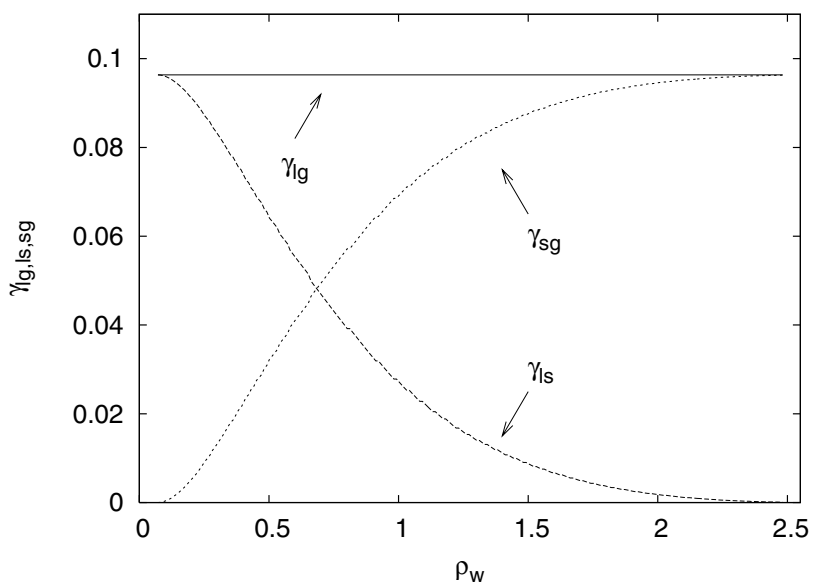

FIG. 2. Surface tensions in a three phase system for $\mathcal{G}_{b}=-6.0$ as a function of $\rho_{w}$. The horizontal line represents the surface tension between liquid and gas $\left(\gamma_{l g}\right)$. The surface tension between solid and gas $\left(\gamma_{s g}\right)$ is zero for $\rho_{w}=\rho_{g}=0.07$ and equal to $\gamma_{l g}$ for $\rho_{w}=\rho_{l}$ $=2.65$. The surface tension between liquid and solid $\left(\gamma_{l s}\right)$ reflects the opposite behavior.

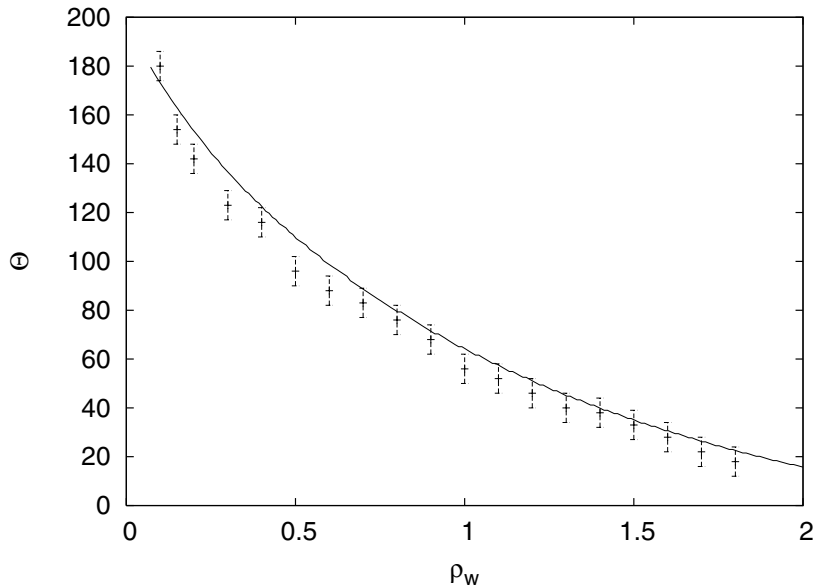

FIG. 3. Contact angle for the case $\mathcal{G}_{b}=-6.0$ as a function of $\rho_{w}$. The analytical estimate is compared to the numerical results of lattice Boltzmann simulations $(+)$. The lattice Boltzmann simulations are carried out in a $2 d$ domain periodic along the stream-wise direction and with two walls (upper and lower wall). The grid mesh used is $L_{x} \times L_{y}=80 \times 45$ and the relaxation time chosen is $\tau=1$. To produce the steady state with a drop of liquid the system has been initialized with a nonhomogeneous condition with a square spot of liquid on the lower wall.

In figure 3 we show the analytical results obtained by inserting the density profile from (24) into (32) and (33). In the same figure we also show the numerical results obtained by running the LBE code and with the estimate of the contact angle obtained with a goniometer as shown in Fig. 4. As one can see from Fig. 3, the method is able to reproduce very hydrophobic material, $\theta \sim 180^{\circ}$ and perfectly wetted surface $\theta \sim 0^{\circ}$, the latter case is obtained when $\rho_{w}$ is chosen equal to the liquid density at the given temperature. All numerical results are obtained by using the shape (20) for the interparticle potential. This is the first study, to our knowledge, where a systematic analytical procedure to derive the contact angle in lattice Boltzmann models with interparticle potentials has been proposed.

The production of a rarefaction zone close to the wall is also important to determine the slippage properties in the case when a constant external pressure drop is applied on the system (Poiseuille flow). For instance, the formation of a gas layer is believed to be the most probable cause of the apparent slip length measured in many experimental microchannels (see [57]). The idea is that the rarefaction layer leads to two feedback on the bulk fluid velocity. First, it allows for the fluid to slide on it without touching the boundary. Second, it gives an effective reduction on the channel width seen by the fluid, leading to an overall increase of the mass throughput for a given pressure drop. Once the density profile is determined, one may solve the equations for the stream-wise velocity (15) profile in presence of a unitary external pressure gradient, $\nabla P_{\text {ext }}$, and a unitary kinematic viscosity, $\nu$, in the Stokes approximation:

$$
\partial_{y}\left(\rho \partial_{y} u_{x}\right)=\frac{\nabla P_{e x t}}{\nu}=1 .
$$

In Fig. 5 we show a few examples of the velocity profile at changing the contact angle. All data have been obtained by 

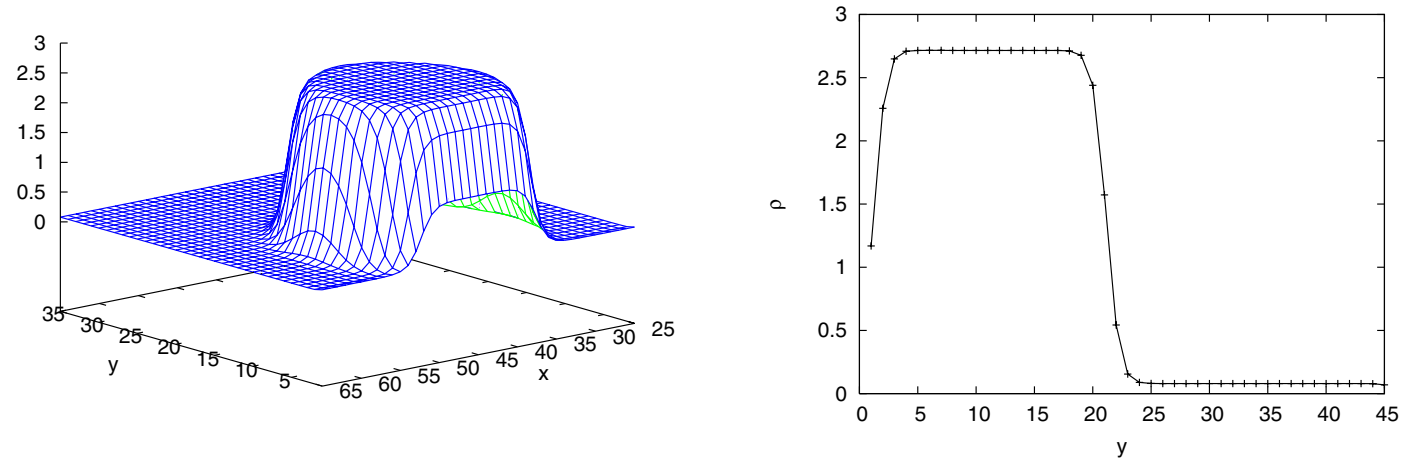

FIG. 4. (Color online) Left: typical density profile from which we extract the contact angle. The contact angle in the numeric is extracted by estimating the angle made by the surface where the density profile is equal to the average value $\left(\rho_{l}-\rho_{g}\right) / 2$ being $\rho_{l}=2.65$ and $\rho_{g}$ $=0.07$ for the present case with $\mathcal{G}_{b}=-6.0$. The computational parameters are the same used as for Fig. 3 . To produce the three phases equilibrium the system has been initialized to a nonhomogeneous condition with a square spot of liquid in contact with the lower wall. Right: a vertical snapshot of the left panel for $x=45$.

imposing that the flow has the fluid density $\left(\rho_{l}\right)$ at the center of the channel and that it ends with a density $\left(\rho_{w}\right)$ at the boundary. Note that, by increasing the hydrophobic property of the surface (increasing $\theta$ ), the rarefaction layer becomes more and more singular, i.e., the velocity profile develops higher and higher shear rates at the boundary.

To quantify this effect, one usually introduces an apparent slip length, $\lambda_{s}$ defined as the length were the bulk, Poiseuillelike, velocity profile extrapolates to zero away from the wall, see Fig. 6. A simple phenomenological estimate of $\lambda_{s}$ for small density variations has been proposed previously in [58] where the authors show that the apparent slip length can be estimated as

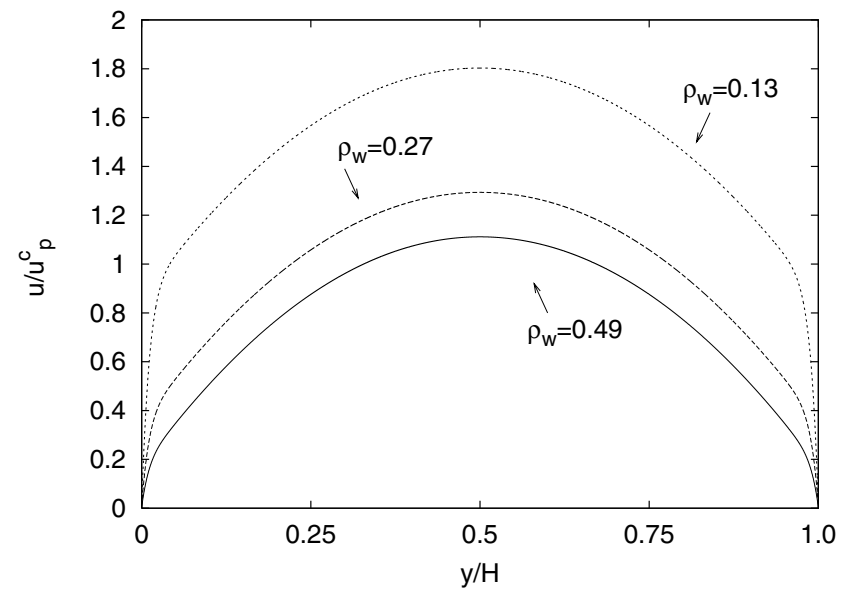

FIG. 5. Velocity profiles for pressure driven Stokes flow. Profiles are obtained integrating numerically the Stokes equation for unit pressure drop and kinematic viscosity (34) in a homogeneous (in the stream-wise direction) channel with a height $L=50$ grid points. The profiles are then normalized with respect to the center channel velocity of the Poiseuille flow $\left(u_{c}^{p}\right)$ for the same geometry. From top to bottom we show different values of $\rho_{w}$ corresponding to different contact angles: $\rho_{w}=0.13 \quad\left(\theta=166^{\circ}\right), \rho_{w}=0.27 \quad(\theta$ $\left.=145^{\circ}\right), \rho_{w}=0.49\left(\theta=110^{\circ}\right)$.
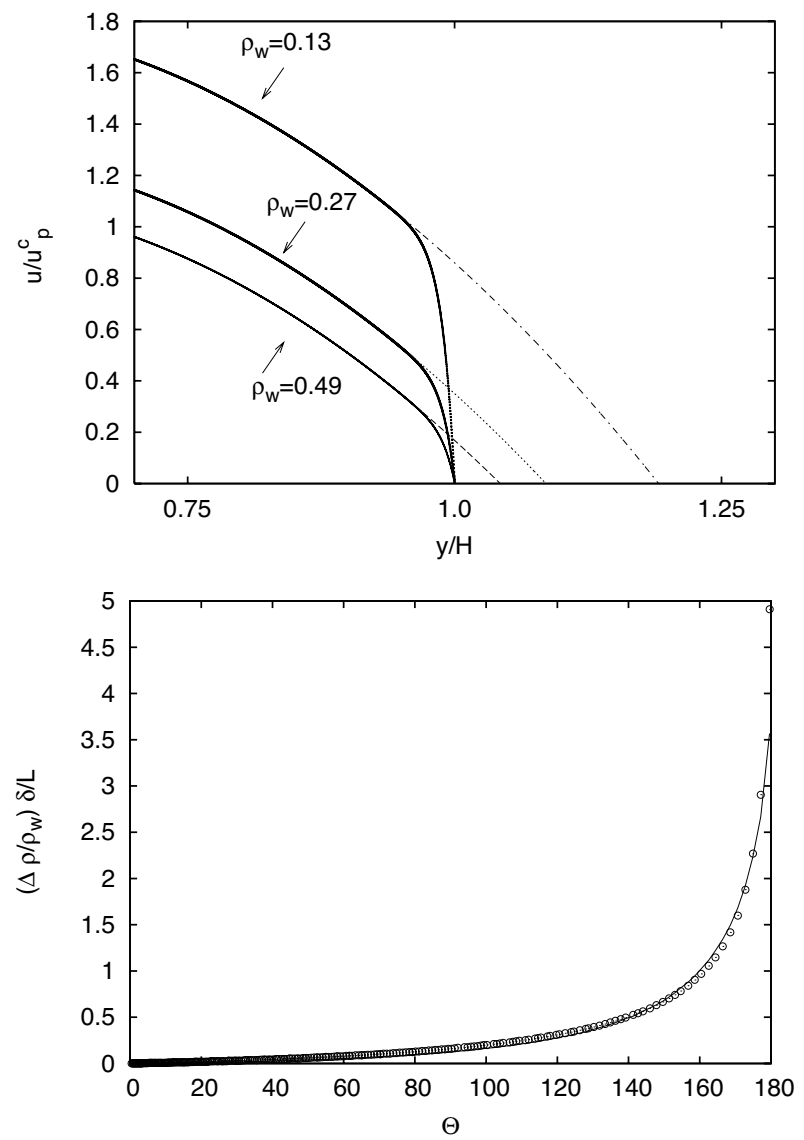

FIG. 6. Slip length for the profiles in the Stokes approximation. In the top panel we show an extrapolation of the profiles shown in the previous figure. The slip length is obtained extrapolating to zero the parabolic profile obtained as a best fit in the bulk region of the channel. In the bottom panel we show the slip length estimated indirectly from the mass flow rate gain with respect to the Poiseuille profile for different contact angles $(\bigcirc)$ and compare to the phenomenological result (35), solid line. The good agreement is obtained with a prefactor in (35) fixed to be 1.2. 


$$
\lambda_{s} \sim \frac{\Delta \rho}{\rho_{w}} \delta,
$$

where with $\Delta \rho$ we mean the density jump from the bulk to the wall values and with $\delta$ is proportional to the rarefaction layer, for a similar study see also [59]. Another important quantity is the mass flow rate gain, i.e., the ratio between the actual mass flow rate and the mass flow rate obtained assuming a perfect Poiseuille-like profile in the whole channel section:

$$
\Phi=\frac{\int d y\left(\rho u_{x}\right)}{L^{3} \nabla P_{e x t} /(12 \nu)},
$$

where $L$ is the total channel height. The mass flow rate is the only quantity which can be easily measured in microdevices experiments. It therefore plays an important role as a benchmark for many modeling methods. In Fig. 6 we show the results for the mass flow rate gain of our mesoscopic model as a function of the contact angle. Note that one can easily gain a factor of the order of $4 \div 5$ times larger for the case of high hydrophobic boundaries. In the same figure (bottom panel) we also show the phenomenological estimate for the mass flow rate gain obtained by using the expression (35) with $\Delta \rho / \rho=\left(\rho_{l}-\rho_{w}\right) / \rho_{w}$ and $\delta$ estimated as the distance (starting from the wall) needed to reach the $98 \%$ of the center channel density (again $\rho_{l}$ ).

\section{Consistency with thermodynamics}

In this section, we want to discuss a few issues on the thermodynamics consistency of the SC model here studied. Let us first start from the simple case of a liquid-gas interface in absence of boundaries. Up to now, in order to get the whole density profile, we have used the mechanical equilibrium condition supplied with the appropriate boundary conditions for the densities in the two phases, see Eqs. (22)-(26). Next, we investigate the relation between this mechanical condition and the thermodynamic conditions on both the density values and on the density profile (i.e., on the surface tension expression). The Maxwell construction which determines the thermodynamics consistency (see also $[60])$ in the $(P, V)$ diagram of the liquid-gas interfaces is built in terms of the requirement that $\int_{\rho_{g}}^{\rho_{l}}\left[P_{b u l k}-P_{b}(\rho)\right] d V$ $=0$ where $V \propto 1 / \rho$. It is easy to realize that this condition leads to the following integral constraint for the two densities, $\rho_{l}, \rho_{g}$, at phase coexistence:

$$
\int_{\rho_{l}}^{\rho_{g}}\left[P_{b u l k}-c_{s}^{2} \rho-\frac{c_{s}^{2} \mathcal{G}_{b}}{2} \psi^{2}(\rho)\right] \frac{1}{\rho^{2}} d \rho=0
$$

which coincides with (26) only if $\psi(\rho) \propto \rho$. This is the indication that the SC choice $\psi(\rho)=\left[1-\exp \left(-\rho / \rho_{0}\right)\right]$ is thermodynamically inconsistent, although one may note that the discrepancy is extremely small in all realistic cases. For instance, in Fig. 7 we show the good agreement of the liquid and gas density values obtained by using the mechanical equilibrium equation (26) and the Maxwell construction (37) for different temperatures $\left(\mathcal{G}_{b}\right)$.

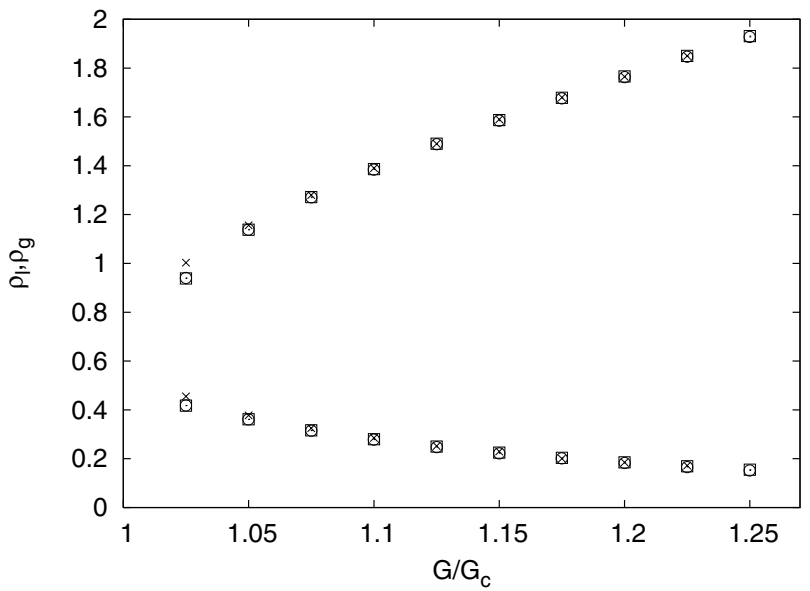

FIG. 7. Results obtained from the mechanical stability conditions (26), $\square$, and thermodynamic coexistence curve (37), $\bigcirc$, for liquid and gas densities. The results of numerical simulation are also reported $(\times)$. The numerical simulations are carried out in a fully periodic $2 d$ setup with grid mesh $L_{x} \times L_{y}=90 \times 90$ and a relaxation time $\tau=1$. The system is then initiated to have a flat interface along the $x$ direction. The top branch refers to the liquid density, $\rho_{l}$, while the bottom branch to the gas density, $\rho_{g}$.

Another problem using the SC approach to describe the interface shape consists in the expression for the surface tension (28) which is proportional to $\left(\partial_{y} \psi\right)^{2}$ instead of being proportional to $\left(\partial_{y} \rho\right)^{2}$ as required by thermodynamical arguments (see [7]). Also in this case, however, the situation is quite encouraging. In fact, let us note that starting from (20) and the expression of the bulk pressure (19), the critical point of the system is identified by the relations

$$
\frac{\partial P_{b}(\rho)}{\partial \rho}=0 ; \quad \frac{\partial^{2} P_{b}(\rho)}{\partial \rho^{2}}=0 .
$$

These are equivalent to

$$
\left(\psi^{\prime}\right)^{2}=-\psi \psi^{\prime \prime} ; \quad \psi \psi^{\prime}=-\frac{1}{\mathcal{G}_{b}} .
$$

Using the functional form $\psi(\rho)=\left[1-\exp \left(-\rho / \rho_{0}\right)\right]$ it is readily checked that

$$
\psi \psi^{\prime \prime}=-\frac{1}{\rho_{0}} \psi \psi^{\prime} .
$$

Now, using (38)-(40) we obtain

$$
\left(\psi^{\prime}\right)^{2}=-\psi \psi^{\prime \prime}=\frac{1}{\rho_{0}} \psi \psi^{\prime}=-\frac{1}{\rho_{0} \mathcal{G}_{b}} .
$$

Since $\psi^{\prime}=\partial \psi / \partial \rho$, this suggests the following scaling relation:

$$
\left|\partial_{y} \psi\right|^{2} \sim-\frac{1}{\mathcal{G}_{b} \rho_{0}}\left|\partial_{y} \rho\right|^{2}
$$

which in turn would imply that the correct matching 


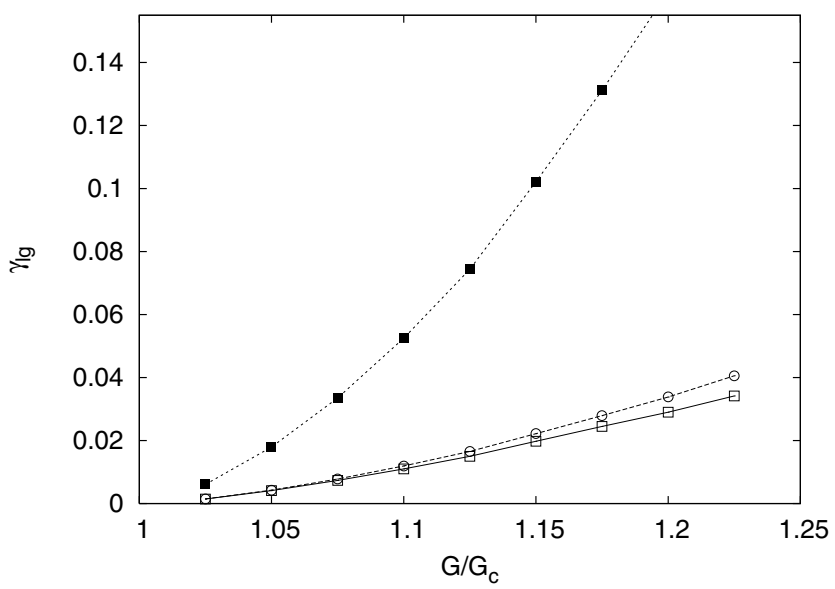

FIG. 8. Surface tension as a function of the interaction parameter $\mathcal{G}_{b}$. The surface tension calculated using both expressions in Eq. (43), the case with $\partial_{y} \rho(\bigcirc)$ and the case with $\partial_{y} \psi(\square)$. To show the importance of the normalization factor (42) we also show the surface tension calculated by simply replacing $\partial_{y} \psi \rightarrow \partial_{y} \rho$ in (28) (filled squares).

$$
\gamma_{l g}=-\frac{1}{2} c_{s}^{4} \mathcal{G}_{b} \int_{-\infty}^{+\infty}\left|\partial_{y} \psi\right|^{2} d y \sim \frac{c_{s}^{4}}{2 \rho_{0}} \int_{-\infty}^{+\infty}\left|\partial_{y} \rho\right|^{2} d y .
$$

The previous argument, although exact at the critical point $\left(\mathcal{G}_{b}=\mathcal{G}_{c}\right)$, is semiquantitatively correct for $\left|\mathcal{G}_{b}\right|>\left|\mathcal{G}_{c}\right|$. In fact, in Fig. 8 we show the comparison between the surface tension measured in our LBE approach and the one defined by (43). The agreement is quite satisfactory for all values of temperature, showing that the model is not far from being consistent also on that side.

\section{E. Two-phase mesoscopic model vs Navier-Stokes equations}

In this section we discuss the interplay between the mesoscopic two-phase approach here proposed in the presence of boundaries and the most traditional "fully macroscopic" description at the hydrodynamical level with ad hoc boundary conditions. A similar study has already been proposed by [22] where a comparison between microscopic MD and macroscopic Stokes flows with slip boundary conditions was presented. In our case, we focus on two analytical results obtained for Navier-Stokes equations in a channel with longitudinal or transverse (with respect to the flow direction) free-slip strips, i.e., the case when some inhomogeneous material is deposited at the surface so as to drastically change the boundary conditions of the Navier-Stokes field from noslip, $\boldsymbol{u}_{\|}=0$, to free-slip, $\partial_{n} \boldsymbol{u}_{\|}=0$ (see Fig. 9). Here $\boldsymbol{u}_{\|}$stands for the velocity component along the surface and $\partial_{n}$ is the derivative along the surface normal direction.

This problem can be attacked at a purely hydrodynamical level, bypassing completely the chemical-physical reactions at the wall that generates these two different boundary conditions and focusing only on the bulk liquid incompressible phase. Using conformal mapping on plane surfaces made up of longitudinal strips [2] proposed an exact analytical solution for the Stokes problem and only very recently a similar
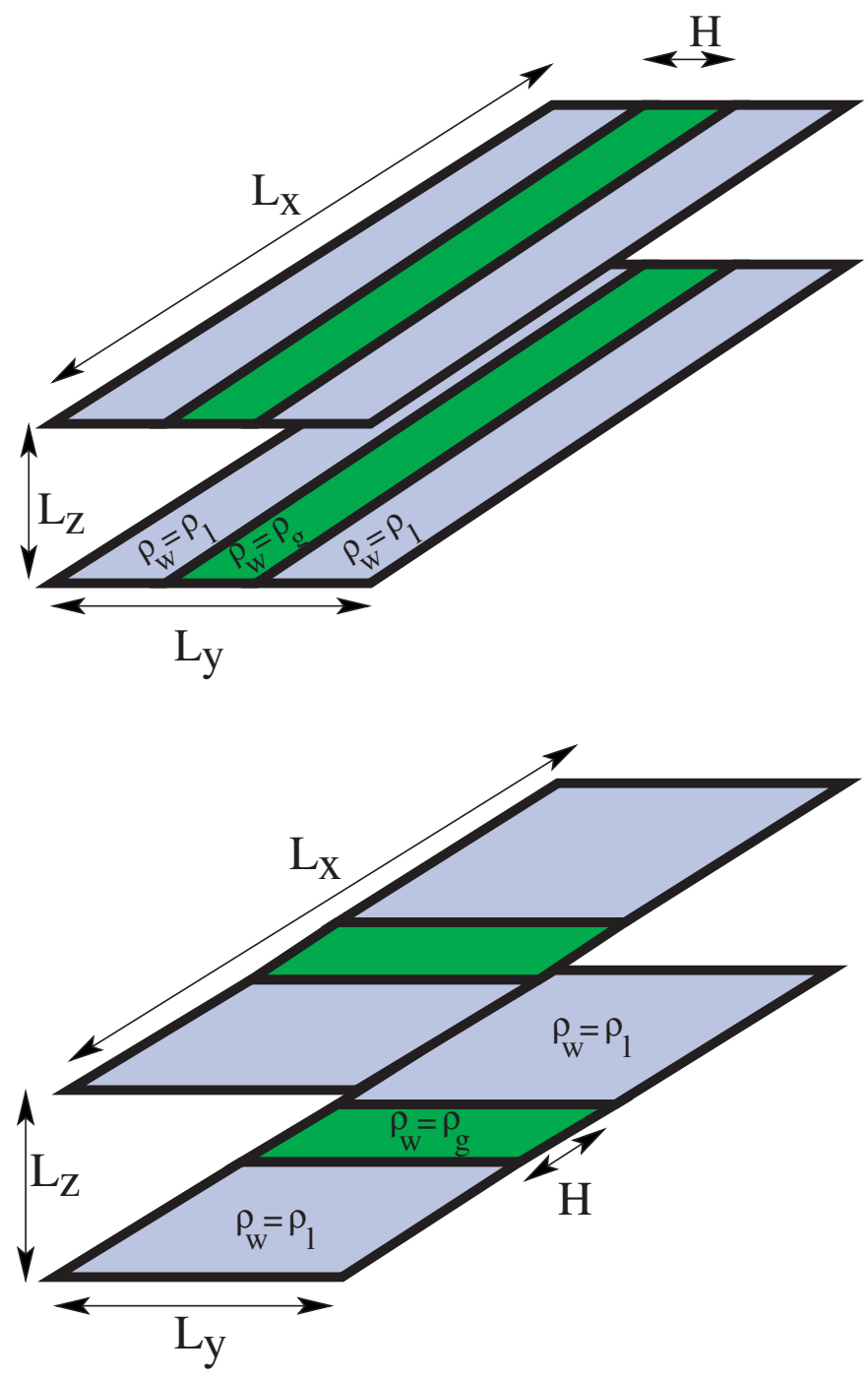

FIG. 9. (Color online) Schematic configurations for the simulations of laminar flows with boundary conditions of free shear and no slip. The alternating pattern of longitudinal (top) and transverse (bottom) strips of free shear is produced by varying the boundary density from complete wetting $\left(\rho_{w}=\rho_{l}\right.$, contact angle $0^{\circ}$, no slip) and perfect dewetting in a strip $H\left(\rho_{w}=\rho_{g}\right.$, contact angle $180^{\circ}$, free shear). With these setups the "slip" probability is $\xi=H / L$ being $L$ $=L_{x}\left(L_{y}\right)$ for transverse (longitudinal) strips. All the details of numerical simulations are given in Fig. 10.

calculation has been proposed for transverse strips [20]. In a previous paper we have shown that the same analytical results can be obtained within the realm of LBE for single flows, but with properly modified boundary conditions [61]. The motivations were very close to the Navier-Stokes continuum approach: neglect microscopic details very close to the boundary, including the possible presence of a rarefaction layer and try to mimic the bulk profile by renormalizing the fluid-wall interactions into a suitable wall function. Next we show that indeed one can recover both the analytical results of $[19,20]$ and the numeric of the single-phase LBE, by using the present two-phase model. In this way, we fill the gap between the bulk physics and the boundary layer physics in 
the proximity of a chemically active wall and we are able to follow also the dynamics inside this latter layer. To accomplish this goal, we performed LBE simulations of the SC model in a channel where $\psi\left(\rho_{w}\right)$ had a periodic structure with alternating strips of hydrophobic $\left(\rho_{w}=\rho_{g}\right.$, local contact angle $\left.180^{\circ}\right)$ and hydrophilic $\left(\rho_{w}=\rho_{l}\right.$, local contact angle $\left.0^{\circ}\right)$ materials. In Fig. 10 (top panel) we show the analytical results for the effective slip length $\lambda_{s}$ obtained (for both longitudinal and transverse strips) by means of the Navier-Stokes (NS) approach and by the present LBE-SC method. The agreement between the two is very good. In the bottom panel, in order to capture the meaning of local boundary conditions obtained using alternating patterns of wetting and nonwetting strips, we compare our multiphase mesoscopic approach to the integration of the incompressible lattice Boltzmann equation with alternating strips of no-slip and free shear [61]. As we can see, while the nonwetting strips simply reduce to a parabolic Poiseuille profile, the presence of the nonwetting strips acts as a free-shear zone where, due to the presence of the gas, the liquid can slide away producing the local freeshear condition.

\section{F. Interaction with the wall: The role of the density gradient}

Up to now we have modeled the wall properties using a single parameter, $\psi\left(\rho_{w}\right)$, which fixes the density of the flow at the boundary. These parameters can be intended as the counterpart of the interaction energy between solid-liquid molecules in an atomistic approach. In principle, also the typical interaction length may play a crucial role, the existence of a characteristic distance is the result of the interplay between attractive and repulsive terms in the Lennard-Jones potential. In order to mimic this effect, it has been proposed to enrich the mesoscopic LBE by assuming that the interaction with the wall is supplemented by another external force $F_{w}$ normal to the wall and decaying exponentially $[43,62]$ :

$$
F_{w}(\boldsymbol{x})=\mathcal{G}_{w} \rho(\boldsymbol{x}) e^{-\left|\boldsymbol{x}-\boldsymbol{x}_{w}\right| / \eta},
$$

where $\boldsymbol{x}_{w}$ is a vector running along the wall location and $\eta$ the typical length scale of the fluid-wall interaction, also known as the Kac range parameter [62]. Equation (44) has been previously used in literature in connection with a slightly different LBE scheme, to show numerically how the wetting angle depends on the ratio $\mathcal{G}_{w} / \mathcal{G}_{b}$ in the presence of phase coexistence between vapor and liquid [44].

The introduction of an external force, exponentially damped in the bulk of the flow, allows the model to control also the gradient of the density profile at the wall. This is a two parameter model able to fit with high accuracy the value of the density at the wall, through $\rho_{w}$, and the derivative of the density at the wall, $\partial_{y} \rho$, through the $\mathcal{G}_{w}$ term in (44).

For the case with $\mathcal{G}_{w}$, one must modify the structure of the pressure tensor as follows:

$$
\begin{aligned}
P_{i j}= & {\left[c_{s}^{2} \rho+\frac{1}{2} c_{s}^{2} \mathcal{G}_{b} \psi^{2}+\frac{1}{2} c_{s}^{4} \mathcal{G}_{b} \psi \Delta \psi+\frac{1}{4} \mathcal{G}_{b} c_{s}^{4}|\nabla \psi|^{2}\right] \delta_{i j} } \\
& -\frac{1}{2} c_{s}^{4} \mathcal{G}_{b} \partial_{i} \psi \partial_{j} \psi-\delta_{i y} \delta_{j y} \mathcal{G}_{w} \int_{0}^{y} \rho(s) e^{-s / \eta} d s .
\end{aligned}
$$
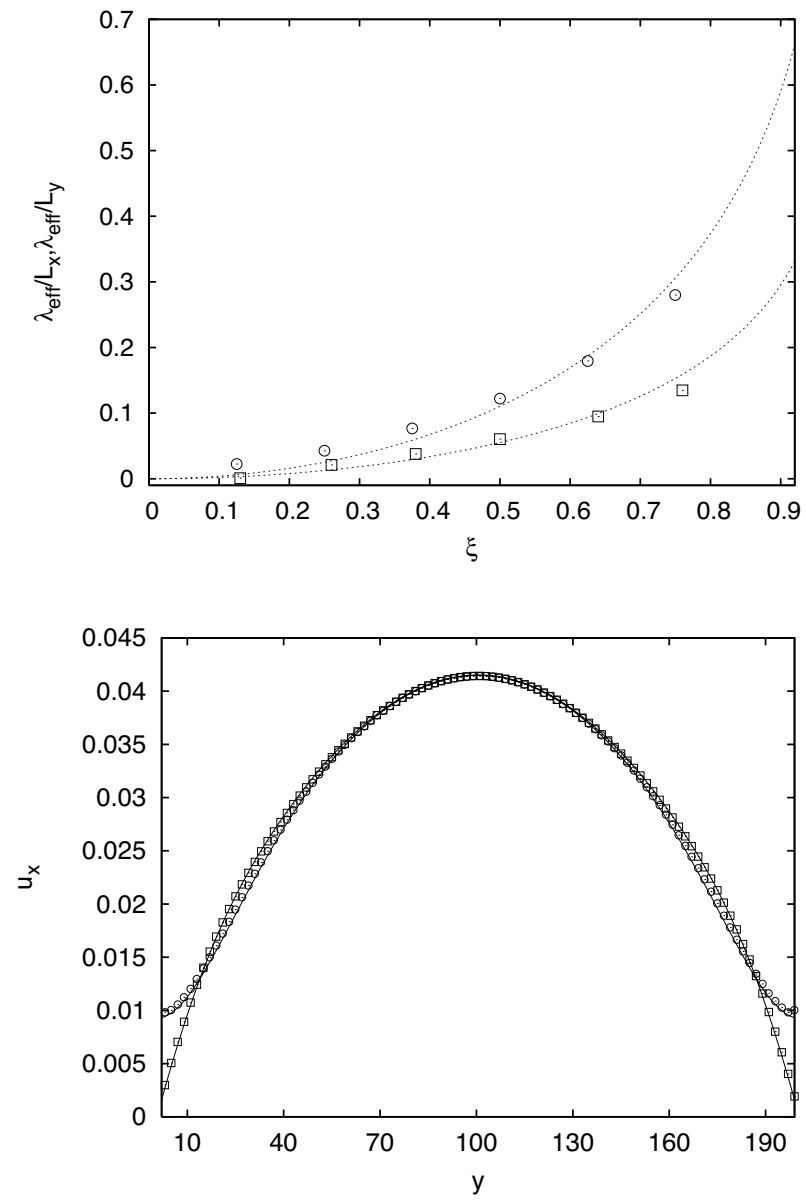

FIG. 10. Effective slip length as a function of the free shear percentage $(\xi)$ in a multiphase approach with alternating strips of wetting and nonwetting properties. The numerical simulations are carried out in 3D setups with $L_{x} \times L_{y} \times L_{z}=64 \times 64 \times 200$ with periodic boundary conditions in the stream-wise $(x)$ and span-wise directions $(y)$. The interaction parameter is $\mathcal{G}_{b}=-6.0$ and the relaxation time is $\tau=1$. The corresponding liquid and gas densities $\left(\rho_{l}\right.$ $\left.=2.65, \rho_{g}=0.07\right)$ are used to mimic no slip and free shear, respectively. Then, the steady state effective slip lengths for transverse $(\square)$ and longitudinal $(\bigcirc)$ strips normalized to the pattern dimension ( $L_{x}$ for transverse and $L_{y}$ for longitudinal) are compared with exact analytical estimates given for stokes flow with alternating non slip and free shear (lines). Bottom panel: The velocity profile in the inlet of the channel and in the middle of a free shear strip from the integration of the incompressible lattice Boltzmann equation with mixed boundary conditions (straight lines) are compared with the results of the mesoscopic multiphase approach ( $\square$ in the inlet and $\bigcirc$ in the middle of free shear strip). The incompressible lattice Boltzmann equation has been used with the same relaxation time and an average density equal to the one of the mesoscopic approach. Both simulations have been forced with a constant pressure gradient so as to reproduce a center channel velocity equal to $u_{c}$ $=0.04$ in LB units.

The off diagonal term, $P_{x y}$, remains the same, while the mismatch between the pressure tensor parallel to the interface, $P_{x x}$ and the pressure term perpendicular to the interface, $P_{y y}$ is changed to 


$$
P_{x x}=P_{y y}+\frac{c_{s}^{4}}{4} \mathcal{G}_{b} \psi \partial_{y} \partial_{y} \psi+\mathcal{G}_{w} \int_{0}^{y} \rho(s) e^{-s / \eta} d s .
$$

This implies that the previous estimate (32) of the contact angle must be replaced by

$$
\cos (\theta)=\frac{\int_{s g}\left(\left|\partial_{y} \psi\right|^{2}+\frac{2 \mathcal{G}_{w}}{\mathcal{G}_{b} c_{s}^{4}} \int_{0}^{y} \rho(s) e^{-s / \eta} d s\right) d y-\int_{s l}\left(\left|\partial_{y} \psi\right|^{2}+\frac{2 \mathcal{G}_{w}}{\mathcal{G}_{b} c_{s}^{4}} \int_{0}^{y} \rho(s) e^{-s / \eta} d s\right) d y}{\int_{l g}\left|\partial_{y} \psi\right|^{2} d y} .
$$

The physics at the boundary now depends on two parameters, which may change the contact angle and the density profile independently. Unfortunately it is very difficult to make any quantitative measurement of the density profile in experiments or MD simulations. It is, therefore, difficult to assess in a systematic way the potential of the LBE model. In Fig. 11 we show the contact angle that can be measured and calculated using (47) in our LBE approach. In the same figure (bottom panel) we also show the variation in the density profile for a few values of the couple $\left(\mathcal{G}_{w}, \rho_{w}\right)$ leading to the same contact angle. As one can see, the model is very sensitive to different choices of the two free parameters, which makes it potentially useful to describe physical situations with large variations in the density profiles in the proximity of the boundaries.

\section{CONCLUSIONS AND PERSPECTIVES}

We have presented a mesoscopic model, based on the Boltzmann equations, for the interaction between a solid wall and a nonideal fluid. The model is an extension of the SC model for dense fluids in unbounded domains. We have first derived an analytical expression for the contact angle and for the surface energy between any two of the liquid, solid, and vapor phases, by introducing a parameter, $\psi\left(\rho_{w}\right)$ which fixes the density value of the Boltzmann molecules at the solid wall. We have shown how in this way one can cover the whole range of contact angles, from a superhydrophobic surface $\theta \sim 180^{\circ}$ to a condition of perfect wettability $\theta \sim 0^{\circ}$. Concerning the thermodynamic consistency of the model we have shown that although not formally verified it does not introduce any systematic error in the results for the surface tension and for the liquid-gas density variations at changing the system temperature (Maxwell construction). We have discussed the connection between the rarefaction layer in the proximity of the wall and the production of an apparent slip phenomenon. We have also presented a comparison between the results obtained within the realm of our two-phase mesoscopic model and some analytical expression for the slip length calculated within a Navier-Stokes approach of a single phase fluid with alternating boundary conditions formed of free-shear and no-slip strips. We have shown that the LBE approach is able to reproduce quantitatively the analytical results, supporting the statement that slip phenomena at the macroscopic Navier-Stokes level can be interpreted by an apparent slip induced by gas accumulation close to the boundary.
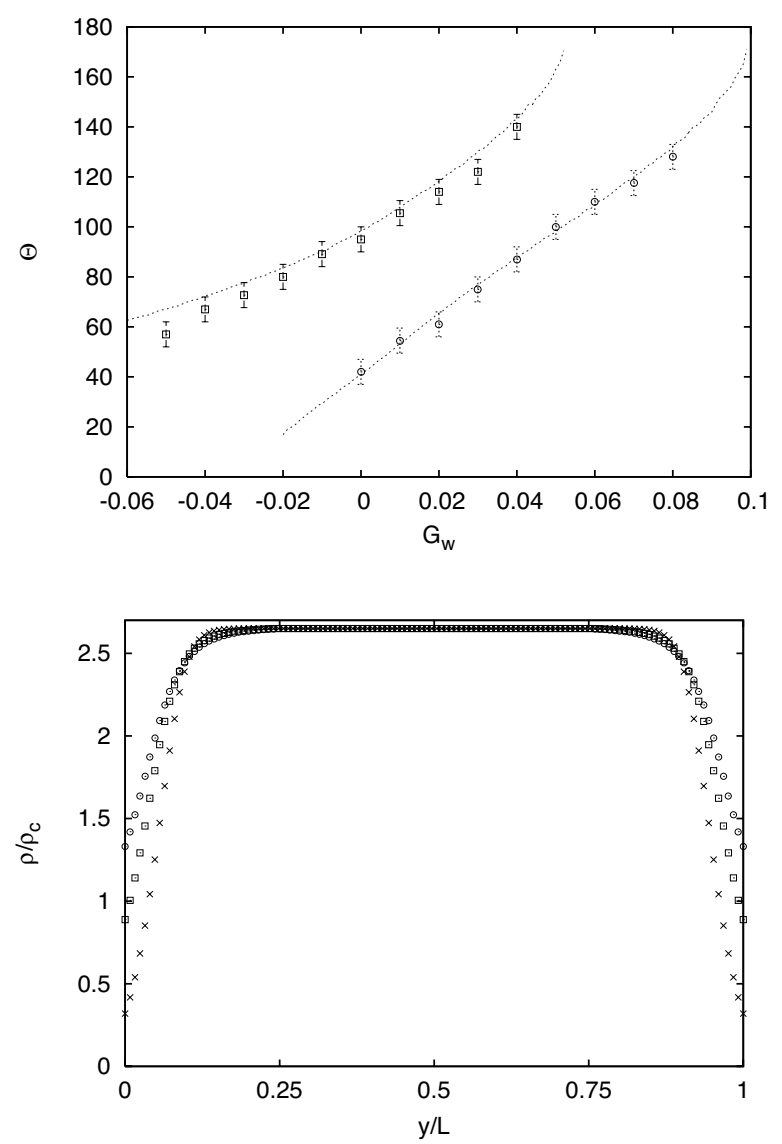

FIG. 11. Contact angle for a two parameter model. Top panel: for a given value of $\rho_{w}$ we show the contact angle for different values of $\mathcal{G}_{w}: \rho_{w}=1.3(\bigcirc), \rho_{w}=0.6(\square)$. The analytical estimate using the mechanical stability is compared with lattice Boltzmann simulations. The details of the numerical simulations are the same for Fig. 3 with the introduction of a wall-fluid force as in (44). Bottom panel: for the same contact angle $\left(130^{\circ}\right)$ we report different profiles obtained for different choices of the parameters $\left(\rho_{w}, \mathcal{G}_{w}\right)$. In particular, the value of $\rho_{w}$ chosen is $\rho_{w}=1.3(\bigcirc) \rho_{w}=0.85(\square) \rho_{w}$ $=0.3(\mathrm{x})$ and the corresponding value of $\mathcal{G}_{w}$ has been chosen to reproduce the same contact angle of $130^{\circ}$ estimated from (47). 
We have also studied the effects on the contact angle and on the density profiles of the use of a second free parameter, $\mathcal{G}_{w}$ connected to the wallforce decaying exponentially inside the bulk of the flow. This second parameter plays an important role if one wants to control both the value of the density at the wall and its gradient.

An important outcome of this study is the possibility to fully integrate the two-phase approach with complex geometries (see also [46]). For instance, recent MD studies [21] have demonstrated the existence of a wetting and/or dewetting transition in microchannels with grooves. The effect is driven by capillarity forces which may expel the liquid out of the corrugation leading to an increase of the effective slip length and of the mass flow rate. The mesoscopic model here presented is fully capable to reproduce the same effects. Moreover, it may also implement more complex surface patterns (different corrugations) than those possible with MD simulations maintaining a control on the spatial and temporal fluctuations at the macroscopic scale. Results on this direction will be reported elsewhere [63].

\section{ACKNOWLEDGMENTS}

We acknowledge useful discussions with D. Lohse who suggested to perform the comparison with the Navier-Stokes equations in the case of longitudinal and transverse free-slip strips. We also thank X. Shan for stimulating discussions.

\section{APPENDIX A}

In this Appendix we detail the calculation needed to perform the continuum limit for the nonideal pressure term. Let us first note that the value of $\Delta t$ just enters the discretized LBE equation (14) as a normalizing factor with respect to the relaxation time $\tau$. Changing $\Delta t$ just means to redefine $\tau$.

Now, if we start from the expression for the $i$ component of the interparticle force

$$
F_{i}(\boldsymbol{x}, t)=-\mathcal{G}_{b} \psi(\boldsymbol{x}) \sum_{\alpha} w_{\alpha} \psi\left(\boldsymbol{x}+\boldsymbol{c}_{\alpha} \Delta t, t\right) c_{\alpha}^{i}
$$

assuming a stationary state $[\boldsymbol{F}(\boldsymbol{x}, t)=\boldsymbol{F}(\boldsymbol{x})]$ and Taylor expanding up to the third order we obtain

$$
\begin{aligned}
F_{i}(\boldsymbol{x})= & -\mathcal{G}_{b} \psi(\boldsymbol{x})\left[\sum_{\alpha} w_{\alpha} c_{\alpha}^{i} \psi(\boldsymbol{x})+\Delta t \sum_{\alpha, j} w_{\alpha} c_{\alpha}^{i} c_{\alpha}^{j} \partial_{j} \psi(\boldsymbol{x})\right. \\
& +\frac{(\Delta t)^{2}}{2} \sum_{\alpha, j, k} w_{\alpha} c_{\alpha}^{i} c_{\alpha}^{j} c_{\alpha}^{k} \partial_{j} \partial_{k} \psi(\boldsymbol{x}) \\
& \left.+\frac{(\Delta t)^{3}}{6} \sum_{\alpha, j, k, l} w_{\alpha} c_{\alpha}^{i} c_{\alpha}^{j} c_{\alpha}^{k} c_{\alpha}^{l} \partial_{j} \partial_{k} \partial_{l} \psi(\boldsymbol{x})\right] .
\end{aligned}
$$

If we choose the weights $w_{\alpha}$ to satisfy the following tensor relations:

$$
\begin{gathered}
\sum_{\alpha} w_{\alpha} c_{\alpha}^{i}=0, \\
\sum_{\alpha} w_{\alpha} c_{\alpha}^{i} c_{\alpha}^{j}=c_{s}^{2} \delta_{i j},
\end{gathered}
$$

$$
\begin{gathered}
\sum_{\alpha} w_{\alpha} c_{\alpha}^{i} c_{\alpha}^{j} c_{\alpha}^{k}=0 \\
\sum_{\alpha} w_{\alpha} c_{\alpha}^{i} c_{\alpha}^{j} c_{\alpha}^{k} c_{\alpha}^{l}=c_{s}^{4}\left(\delta_{i j} \delta_{k l}+\delta_{i k} \delta_{j l}+\delta_{i l} \delta_{j k}\right),
\end{gathered}
$$

we end up with the following expression for the $i$ component of $\boldsymbol{F}$ :

$$
F_{i}=-\mathcal{G}_{b} \psi \Delta t c_{s}^{2} \partial_{i} \psi-\mathcal{G}_{b} \psi \frac{(\Delta t)^{3}}{2} c_{s}^{4} \partial_{i} \Delta \psi
$$

being $\Delta$ the Laplacian operator. The above expression for the interparticle force can be easily translated into an excess pressure with respect to the ideal gas expression $\left(c_{s}^{2} \rho\right)$ using the definition

$$
-\partial_{j} P_{i j}+\partial_{i}\left(c_{s}^{2} \rho\right)=F_{i}
$$

and, therefore, we end up with a pressure tensor of the form:

$$
\begin{aligned}
P_{i j}= & {\left[c_{s}^{2} \rho+\frac{\Delta t}{2} c_{s}^{2} \mathcal{G}_{b} \psi^{2}+\frac{(\Delta t)^{3}}{2} c_{s}^{4} \mathcal{G}_{b} \psi \Delta \psi\right.} \\
& \left.+\frac{\mathcal{G}_{b} c_{s}^{4}(\Delta t)^{3}}{4}|\nabla \psi|^{2}\right] \delta_{i j}-\frac{(\Delta t)^{3}}{2} c_{s}^{4} \mathcal{G}_{b} \partial_{i} \psi \partial_{j} \psi
\end{aligned}
$$

An important remark is now in order. The continuum expression just derived depends explicitly on the interparticle potential range, here of the order of $c_{s} \Delta t$. The limit $\Delta t \rightarrow 0$ would, therefore, imply a vanishing interaction range, i.e., the ideal gas limit $P_{i j}=c_{s}^{2} \rho \delta_{i j}$.

\section{APPENDIX B}

In this Appendix we will detail the calculation of the contact angle used throughout the text. Starting from the pressure tensor (for $\Delta t=1$ )

$$
\begin{aligned}
P_{i j}= & {\left[c_{s}^{2} \rho+\frac{1}{2} c_{s}^{2} \mathcal{G}_{b} \psi^{2}+\frac{1}{2} c_{s}^{4} \mathcal{G}_{b} \psi \Delta \psi+\frac{1}{4} \mathcal{G}_{b} c_{s}^{4}|\nabla \psi|^{2}\right] \delta_{i j} } \\
& -\frac{1}{2} c_{s}^{4} \mathcal{G}_{b} \partial_{i} \psi \partial_{j} \psi .
\end{aligned}
$$

In the coordinate system of Fig. 12 we make the following change of variables:

$$
\begin{aligned}
& x^{\prime}=+x \sin (\theta)+y \cos (\theta) \\
& y^{\prime}=-x \cos (\theta)+y \sin (\theta)
\end{aligned}
$$

being $y^{\prime}$ aligned with the separation line between liquid and gas.

$$
\begin{aligned}
& \frac{\partial}{\partial x}=\frac{\partial x^{\prime}}{\partial x} \frac{\partial}{\partial x^{\prime}}+\frac{\partial y^{\prime}}{\partial x} \frac{\partial}{\partial y^{\prime}}=\sin (\theta) \frac{\partial}{\partial x^{\prime}}-\cos (\theta) \frac{\partial}{\partial y^{\prime}}, \\
& \frac{\partial}{\partial y}=\frac{\partial x^{\prime}}{\partial y} \frac{\partial}{\partial x^{\prime}}+\frac{\partial y^{\prime}}{\partial y} \frac{\partial}{\partial y^{\prime}}=\cos (\theta) \frac{\partial}{\partial x^{\prime}}+\sin (\theta) \frac{\partial}{\partial y^{\prime}} .
\end{aligned}
$$

If we assume only the $x^{\prime}$ dependence we can write

$$
\psi(x, y)=\psi\left(x^{\prime}\right),
$$




$$
\begin{gathered}
\frac{\partial}{\partial x}=\sin (\theta) \frac{\partial}{\partial x^{\prime}}, \\
\frac{\partial}{\partial y}=\cos (\theta) \frac{\partial}{\partial x^{\prime}}, \\
\int d x \rightarrow \frac{1}{\sin (\theta)} \int d x^{\prime},
\end{gathered}
$$

which clearly imply

$$
\begin{aligned}
\int\left(\partial_{x} \psi\right)\left(\partial_{y} \psi\right) d x & =\frac{1}{\sin (\theta)} \int d x^{\prime} \sin (\theta) \cos (\theta)\left(\partial_{x^{\prime}} \psi\right)\left(\partial_{x^{\prime}} \psi\right) \\
& =\int d x^{\prime} \cos (\theta)\left(\partial_{x^{\prime}} \psi\right)\left(\partial_{x^{\prime}} \psi\right) .
\end{aligned}
$$

By imposing a zero flux out of the boundaries of the vector $\left(P_{x x}, P_{x y}\right)$ we obtain the mechanical definition of the contact angle. To this purpose, let us note that based on the definition of the pressure tensor, we can write

$$
\begin{gathered}
P_{x x}=P_{y y}+\frac{c_{s}^{4}}{2} \mathcal{G}_{b} \partial_{y} \psi \partial_{y} \psi, \\
P_{x y}=\frac{c_{s}^{4}}{2} \mathcal{G}_{b} \partial_{x} \psi \partial_{y} \psi,
\end{gathered}
$$

with $P_{y y}$ constant along the solid-liquid and solid-gas interface (segments $A, B$ of Fig. 12). This, together with (58) implies

$$
\begin{gathered}
-\int_{s l} \frac{c_{s}^{4}}{2} \mathcal{G}_{b} \partial_{y} \psi \partial_{y} \psi d y+\int_{s g} \frac{c_{s}^{4}}{2} \mathcal{G}_{b} \partial_{y} \psi \partial_{y} \psi d y \\
-\cos (\theta) \int_{l g} \frac{c_{s}^{4}}{2} \mathcal{G}_{b} \partial_{x^{\prime}} \psi \partial_{x^{\prime}} \psi d x^{\prime}=0
\end{gathered}
$$

and

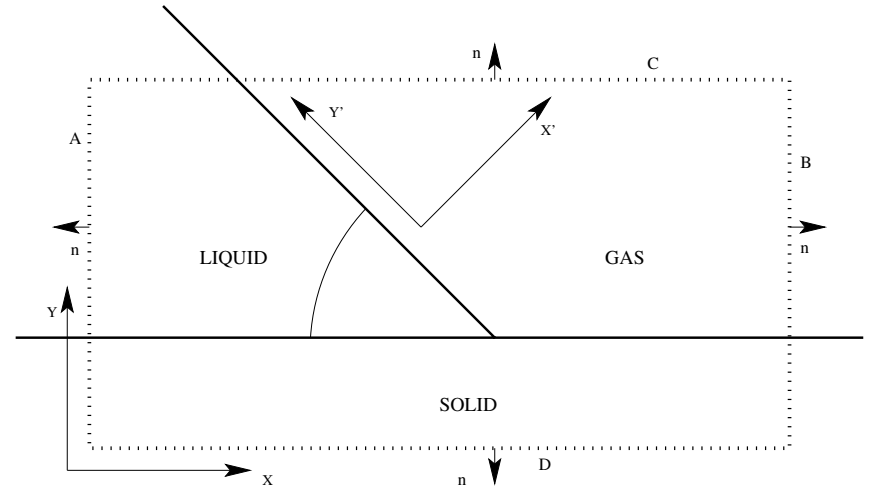

FIG. 12. The schematic figure of the contact angle setup.

$$
\cos (\theta)=\frac{\int_{s g}\left|\partial_{y} \psi\right|^{2} d y-\int_{s l}\left|\partial_{y} \psi\right|^{2} d y}{\int_{l g}\left|\partial_{x^{\prime}} \psi\right|^{2} d x^{\prime}}
$$

For the case with $\mathcal{G}_{w}$, the previously used pressure tensor must be slightly modified due to the presence of a normal (say along the $y$ direction) force between the fluid and the wall:

$$
\begin{aligned}
P_{i j}= & {\left[c_{s}^{2} \rho+\frac{1}{2} c_{s}^{2} \mathcal{G}_{b} \psi^{2}+\frac{1}{2} c_{s}^{4} \mathcal{G}_{b} \psi \Delta \psi+\frac{1}{4} \mathcal{G}_{b} c_{s}^{4}|\nabla \psi|^{2}\right] \delta_{i j} } \\
& -\frac{1}{2} c_{s}^{4} \mathcal{G}_{b} \partial_{i} \psi \partial_{j} \psi-\delta_{i y} \delta_{j y} \mathcal{G}_{w} \int_{0}^{y} \rho(s) e^{-s / \eta} d s
\end{aligned}
$$

Then relations and (B8) and (B9) should be slightly modified since $P_{x y}$ gives the same result but for $P_{x x}$ we have

$$
P_{x x}=P_{y y}+\frac{c_{s}^{4}}{2} \mathcal{G}_{b} \partial_{y} \psi \partial_{y} \psi+\mathcal{G}_{w} \int_{0}^{y} \rho(s) e^{-s / \eta} d s
$$

that imply the following estimate for the contact angle:

$$
\cos (\theta)=\frac{\int_{s g}\left(\left|\partial_{y} \psi\right|^{2}+\frac{2 \mathcal{G}_{w}}{\mathcal{G}_{b} c_{s}^{4}} \int_{0}^{y} \rho(s) e^{-s / \eta} d s\right) d y-\int_{s l}\left(\left|\partial_{y} \psi\right|^{2}+\frac{2 \mathcal{G}_{w}}{\mathcal{G}_{b} c_{s}^{4}} \int_{0}^{y} \rho(s) e^{-s / \eta} d s\right) d y}{\int_{l g}\left|\partial_{y} \psi\right|^{2} d y} .
$$

Note that the importance of wall effects appears only in relation to bulk terms in $\frac{2 \mathcal{G}_{w}}{\mathcal{G}_{b} c_{s}^{4}}$.

[1] G. Whitesides and A. Stroock, Phys. Today 54(6), 42 (2001).

[2] M. Gad-el'Hak, J. Fluids Eng. 121, 5 (1999).

[3] C.-M. Ho and Y.-C. Tai, Annu. Rev. Fluid Mech. 30, 579 (1998).

[4] P. G. de Gennes, Capillarity and Wetting Phenomena: Drops,
Bubbles, Pearls, Waves, Springer, New York, (2003).

[5] P. Tabeling, Introduction á la Microfluidique (Springer, Berlin, 2003).

[6] G. Karnikadis and A. Beskok, Microflows (Springer-Verlag, Berlin, 2002). 
[7] J. S. Rowlinson and B. Widom, Molecular Theory of Capillarity (Clarendon, Oxford, 1982).

[8] J. Bico, C. Marzolin and D. Quere, Europhys. Lett. 47, 220 (1999).

[9] V. S. J. Craig, C. Neto, and D. R. M. Williams, Phys. Rev. Lett. 87, 054504 (2001).

[10] J. Ou, B. Perot, and J. Rothstein, Phys. Fluids 16, 4635 (2004).

[11] J. Maurer, P. Tabeling, P. Joseph, and H. Willaime, Phys. Fluids 15, 2613 (2004).

[12] T. Onda, S. Shibuichi, N. Satoh, and K. Tsujii, Langmuir 12, 2125 (1996).

[13] R. Pit, H. Hervet, and L. Leger, Phys. Rev. Lett. 85, 980 (2000).

[14] O. Vinogradova and G. Yabukov, Langmuir 19, 1227 (2003).

[15] Y. Zhu and S. Granick, Phys. Rev. Lett. 87, 096105 (2001).

[16] Y. Zhu and S. Granick, Phys. Rev. Lett. 88, 106102 (2002).

[17] J.-T. Cheng and N. Giordano, Phys. Rev. E 65, 031206 (2002).

[18] C. Choi, K. Johan, A. Westin, and K. Breuer, Phys. Fluids 15, 2897 (2003).

[19] E. Lauga and H. Stone, J. Fluid Mech. 489, 55 (2003).

[20] J. Philip, Z. Angew. Math. Phys. 23, 353 (1972); 23, 960 (1972).

[21] C. Cottin-Bizonne, C. Barentine, E. Charlaix, L. Bocquet, and J.-L. Barrat, Eur. Phys. J. E 15, 427 (2004).

[22] N. V. Priezjev, A. A. Darhuber, and S. M. Troian, Phys. Rev. E 71, 041608 (2005).

[23] L. Bocquet, and J.-L. Barrat, Phys. Rev. Lett. 70, 2726 (1993).

[24] M. Cieplak, J. Koplik, and J. R. Banavar, Phys. Rev. Lett. 86, 803 (2001)

[25] P. A. Thompson and M. O. Robbins, Phys. Rev. Lett. 63, 766 (1989).

[26] P. A. Thompson and M. O. Robbins, Phys. Rev. A 41, 6830 (1990).

[27] P. A. Thompson and S. M. Troian, Nature (London) 389, 360 (1997).

[28] H. Brenner and V. Ganesan, Phys. Rev. E 61, 6879 (2000).

[29] J. Koplik and J. Banavar, Annu. Rev. Fluid Mech. 27, 257 (1991).

[30] D. Rapaport, The Art of Molecular Dynamics Simulations (Cambridge University Press, Cambridge, 1995).

[31] P. Chapman and Cowling, The Mathemathical Theory of Nonuniform Gases (Cambridge University Press, London, 1970).

[32] C. Cercignani, The Mathematical Theory on Non Uniform Gases: An Account of the Kinetic Theory of Viscosity, Thermal Conduction and Diffusion in Gases (Cambridge University Press, Cambridge, 1991).

[33] G. R. McNamara and G. Zanetti, Phys. Rev. Lett. 61, 2332 (1998).

[34] F. Higuera and J. Jimenez, Europhys. Lett. 9, 663 (1989).
[35] F. Higuera, S. Succi, and R. Benzi, Europhys. Lett. 9, 345 (1989).

[36] S. Chen, and G. Doolen, Annu. Rev. Fluid Mech. 30, 329 (1998)

[37] R. Benzi, S. Succi, and M. Vergassola, Phys. Rep. 222, 145 (1992).

[38] J. Mohling, Statistical Mechanics: Methods and Applications (John Wiley and Sons, New York, 1982).

[39] J. H. Irving and J. G. Kirkwood, J. Chem. Phys. 18, 817 (1950).

[40] X. Shan and H. Chen, Phys. Rev. E 49, 2941 (1994).

[41] X. Shan and H. Chen, Phys. Rev. E 47, 1815 (1993).

[42] M. R. Swift, E. Orlandini, W. R. Osborn, and J. M. Yeomans, Phys. Rev. E 54, 5041 (1996).

[43] X. He and G. Doolen, J. Stat. Phys. 107, 309 (2002).

[44] J. Zhang and D. Kwok, Phys. Rev. E 70, 056701 (2004).

[45] A. J. Briant, A. J. Wagner, and J. M. Yeomans, Phys. Rev. E 69, 031602 (2004).

[46] R. Verberg, C. M. Pooley, J. M. Yeomans, and A. C. Balazs, Phys. Rev. Lett. 93, 184501 (2004).

[47] H. Kusumaatmaja, J. Leopoldes, A. Dupuis, and J. M. Yeomans, Europhys. Lett. 73, 740 (2006).

[48] J. W. Cahn, J. Chem. Phys. 66, 3667 (1997).

[49] J. Zhang, B. Li, and D. Kwok, Phys. Rev. E 69, 032602 (2004).

[50] H. E. Stanley, Introduction to Phase Transitions and Critical Phenomena, International Series of Monographs on Physics (Oxford University Press, Oxford, 1971).

[51] K. Huang, Statistical Mechanics (Wiley, New York, 1987).

[52] Martys, Int. J. Mod. Phys. C 10, 1367 (1999).

[53] X. He, X. Shan, and G. Doolen, Phys. Rev. E 57, R13 (1998).

[54] P. L. Bhatnagar, E. Gross, and M. Krook, Phys. Rev. 94, 511 (1954).

[55] S. Succi, The Lattice Boltzmann Equation (Oxford Science, Oxford, 2001).

[56] D. Wolf-Gladrow, Lattice-Gas Cellular Automata and Lattice Boltzmann Models (Springer, New York, 2000).

[57] D. Tretheway and C. Meinhart, Phys. Fluids 14, L9 (2002).

[58] R. Benzi, L. Biferale, M. Sbragaglia, S. Succi, and F. Toschi, Europhys. Lett. 74, 651 (2006).

[59] J. Harting, C. Kunert, and H. J. Herrmann, Europhys. Lett. 75, 328 (2006).

[60] H. B. Callen, Thermodynamics and an introduction to Thermostatistics (Wiley, New York, 1985).

[61] R. Benzi, L. Biferale, M. Sbragaglia, S. Succi, and F. Toschi, J. Fluid Mech. 548, 257 (2006).

[62] D. E. Sullivan, J. Chem. Phys. 74, 2604 (1981).

[63] R. Benzi, L. Biferale, M. Sbragaglia, S. Succi, and F. Toschi, e-print nlin.CG/0605013. 